File: Jianbo_v1.0-MPEG-IBOA-Supporting information_2016May29

\title{
Alcoholic Photoinitiated Polymerization-Induced Self-Assembly (Photo-PISA): A Fast Route toward Poly(isobornyl acrylate)-based Diblock Copolymer Nano-objects
}

Jianbo Tan ${ }^{* a, b}$, Chundong Huang ${ }^{\mathrm{a}}$, Dongdong Liu ${ }^{\mathrm{a}}$, Xuechao Zhanga ${ }^{\mathrm{a}}$, Yuhao Bai ${ }^{\mathrm{a}}$, Li Zhang ${ }^{\text {a,b }}$

a. Department of Polymeric Materials and Engineering, School of Materials and Energy, Guangdong University of Technology, Guangzhou 510006, China.

b. Guangdong Provincial Key Laboratory of Functional Soft Condensed Matter, Guangzhou 510006, China.

*Corresponding authors: tanjianbo@gdut.edu.cn, lizhang@gdut.edu.cn 


\section{EXPERIMENTAL SECTION}

Materials. Isobornyl acrylate (IBOA, Sigma-Aldrich) was purified by passing through a basic alumina oxide (Aladdin) column prior to storage at $4{ }^{\circ} \mathrm{C}$. Monomethoxy poly(ethylene glycol) ( $\mathrm{mPEG}_{45}, 2000 \mathrm{~g} / \mathrm{mol}$, Sigma-Aldrich), dicyclohexylcarbodiimide (DCC, Aladdin), 4-dimethylaminopyridine (DMAP, Aladdin), hydroquinone (Aladdin), and 2,4,6-trimethylbenzoyldi-phenylphosphinate (TPO, Ciba) were used without further purification. 2, 2-Azobisisobutyronitrile (AIBN, Aladdin) was recrystallized from ethanol prior to storage under refrigeration at $4{ }^{\circ} \mathrm{C}$. S-1-dodecyl-S'-( $\alpha, \alpha^{\prime}$-dimethyl- $\alpha$ "-acetic acid) trithiocarbonate (DDMAT) was synthesized according to a published procedure. ${ }^{1}$

Synthesis of mPEG45-DDMAT. A solution of DDMAT (7.29 g, $20 \mathrm{mmol})$ in $40 \mathrm{~mL}$ of anhydrous dichloromethane (DCM) was introduced in a dry flask under nitrogen atmosphere containing $\mathrm{mPEG}_{45}(20 \mathrm{~g}, 10 \mathrm{mmol})$. Then a solution of DCC (4.12 g, $\left.20 \mathrm{mmol}\right)$ and DMAP $(0.244 \mathrm{~g}, 2 \mathrm{mmol})$ in $10 \mathrm{~mL}$ of anhydrous THF was added dropwise to the reaction mixture at $0{ }^{\circ} \mathrm{C}$. The esterification reaction proceeded under stirring at room temperature for $48 \mathrm{~h}$. The polymer was collected by precipitation of the reaction mixture in cold diethyl ether, and finally dried at $45^{\circ} \mathrm{C}$ under vacuum to obtain a yellow powder.

Photoinitiated RAFT dispersion polymerization. In a typical experiment, IBOA (1.5 g, 7.2 mmol), mPEG 45 -DDMAT $(0.085 \mathrm{~g}, 0.036 \mathrm{mmol})$, and TPO (0.0046 g, $0.012 \mathrm{mmol})$ were weighed into a $25 \mathrm{~mL}$ round bottom flask. Then an ethanol/water mixture $(2.98 \mathrm{~g} / 0.52 \mathrm{~g}, 85 / 15$, w/w) was added into the flask to dissolve all the reagents. The flask was immersed into a 
pre-heated water bath $\left(40{ }^{\circ} \mathrm{C}\right)$, purged with nitrogen for $20 \mathrm{~min}$, and then irradiated by a visible light LED lamp (405 nm, $\left.0.5 \mathrm{~mW} / \mathrm{cm}^{2}\right)$ for $1 \mathrm{~h}$. The reaction was quenched by exposure to air and the addition of a small amount of hydroquinone.

Kinetic study of photoinitiated RAFT dispersion polymerization. IBOA (1.5 g, 7.2 mmol), mPEG 45 -DDMAT $(0.085 \mathrm{~g}, 0.036 \mathrm{mmol})$, and TPO (0.0046 g, $0.012 \mathrm{mmol})$ were weighed into a $25 \mathrm{~mL}$ round bottom flask. Then an ethanol/water mixture $(2.98 \mathrm{~g} / 0.52 \mathrm{~g}, 85 / 15$, w/w) was added into the flask to dissolve all the reagents. The flask was put into a pre-heated water bath $\left(40{ }^{\circ} \mathrm{C}\right)$, purged with nitrogen for $20 \mathrm{~min}$, and then irradiated by a visible light LED lamp $\left(405 \mathrm{~nm}, 0.5 \mathrm{~mW} / \mathrm{cm}^{2}\right)$. Samples were withdrawn at predetermined time intervals by syringes under nitrogen, and the reaction was quenched by exposure to air and the addition of a small amount of hydroquinone. The samples were then analysis by ${ }^{1} \mathrm{H}$ NMR spectroscopy and GPC.

Thermally initiated RAFT dispersion polymerization. In a typical experiment, IBOA (1.5 g, $7.2 \mathrm{mmol}), \mathrm{mPEG}_{45}$-DDMAT (0.085 g, $\left.0.036 \mathrm{mmol}\right)$, and AIBN (0.002 g, $\left.0.012 \mathrm{mmol}\right)$ were weighed into a $25 \mathrm{~mL}$ round bottom flask. Then an ethanol/water mixture $(2.98 \mathrm{~g} / 0.52 \mathrm{~g}, 85 / 15$, w/w) was added into the flask to dissolve all the reagents. The reaction was purged with nitrogen for 20 min, and then immersed into a pre-heated oil bath at $70^{\circ} \mathrm{C}$. The reaction was quenched by immersing into an ice-water bath.

Kinetic study of thermally initiated RAFT dispersion polymerization. IBOA (1.5 g, 7.2 mmol), $\mathrm{mPEG}_{45}$-DDMAT $(0.085 \mathrm{~g}, 0.036 \mathrm{mmol})$, and AIBN (0.002 $\left.\mathrm{g}, 0.012 \mathrm{mmol}\right)$ were 
weighed into a $25 \mathrm{~mL}$ round bottom flask. Then an ethanol/water mixture $(2.98 \mathrm{~g} / 0.52 \mathrm{~g}, 85 / 15$, w/w) was added into the flask to dissolve all the reagents. The reaction was purged with nitrogen for $20 \mathrm{~min}$, and then immersed into a pre-heated oil bath at $70^{\circ} \mathrm{C}$. Samples were withdrawn at predetermined time intervals by syringes under nitrogen, and the reaction was quenched by immersing into an ice-water bath and the addition of a small amount of hydroquinone. The samples were then analysis by ${ }^{1} \mathrm{H}$ NMR spectroscopy and GPC.

Characterization. The polymerization reaction mixtures were diluted 100-fold with ethanol-water mixtures. A drop of the solution was placed on the copper grip for 1 min and then blotted with filter paper to remove excess solution. A drop of uranyl acetate solution (0.5 wt \%) was soaked on the same copper grip for $1.5 \mathrm{~min}$, and then blotted with filter paper to remove excess strain. Transmission electron microscope (TEM) observations were carried out on a FEI Tecnai G2 Spirit instrument operated at $120 \mathrm{kV}$.

The molecular weight and polydispersity of the block copolymers were measured by gel permeation chromatography (GPC) at $40{ }^{\circ} \mathrm{C}$ using a Waters $1515 \mathrm{GPC}$ instrument with tetrahydrofuran (THF) as the mobile phase and Waters styragel HR1, HR4 columns. The flow rate of THF was $1 \mathrm{~mL} / \mathrm{min}$. Linear poly (methyl methacrylate) polymers with narrow molecular weight distributions were used as the standards to calibrate the apparatus.

${ }^{1} \mathrm{H}$ NMR spectra were recorded in $\mathrm{CDCl}_{3}$ using a Bruker Avance III $400 \mathrm{MHz} \mathrm{NMR}$ spectrometer at a temperature of $25{ }^{\circ} \mathrm{C}$. Note: The samples prepared by PISA were first diluted with methanol, and then dissolved in $\mathrm{CDCl}_{3}$. 
UV-Visible spectra were recorded with a $1.0 \mathrm{~cm}$ quartz cuvette using a UV2450 spectrometer.

Intensity-average hydrodynamic diameters of the nanaoparticles $(0.1 \% \mathrm{v} / \mathrm{v})$ were obtained in water using a Brookhaven nanoparticle Size-Zeta potential and molecular weight analyzer.

\section{ADDITIONAL RESULTS}
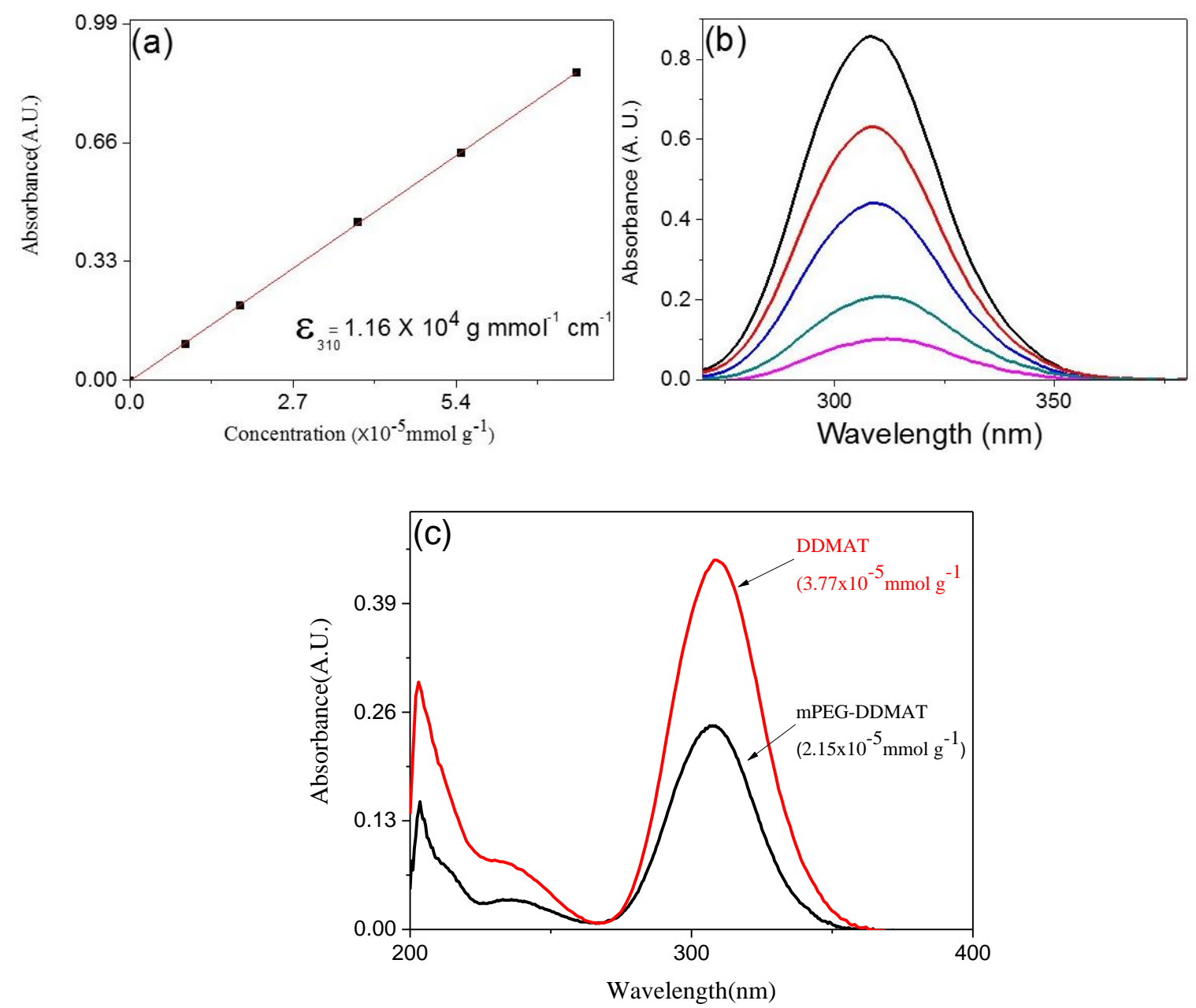

Figure S1. (a) Beer-Lambert linear calibration plot obtained for DDMAT, (b) UV-vis absorption spectra recorded for DDMAT at different concentrations as Figure S1a, (c) UV-vis absorption spectra of mPEG 45 -DDMAT and the DDMAT precursor. 


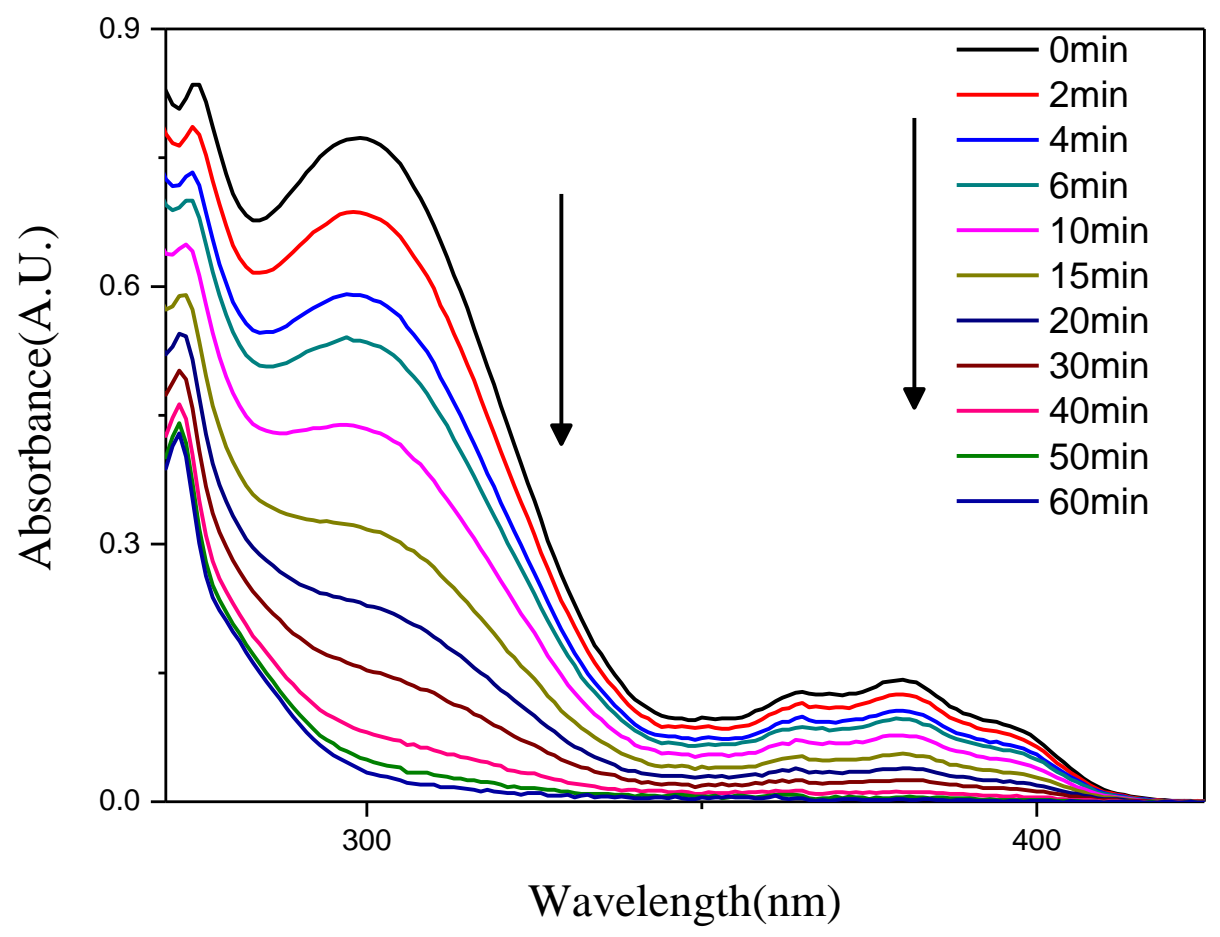

Figure S2. UV-vis absorption spectra recorded for the decomposition of TPO in ethanol-water $(85 / 15, w / w)$ under the same conditions of photo-PISA.
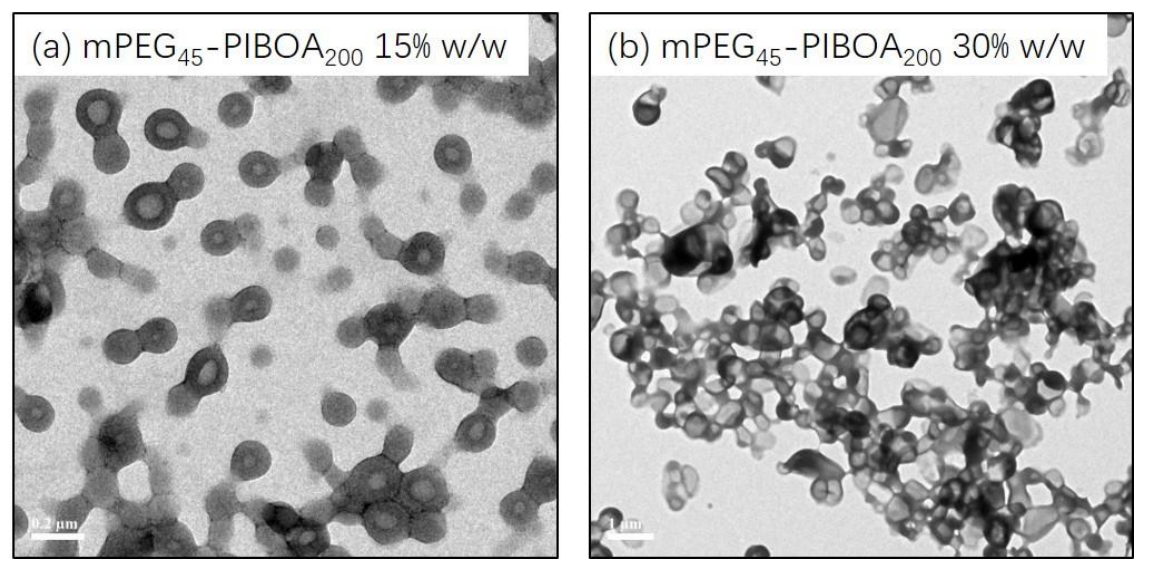

Figure S3. TEM images of $\mathrm{mPEG}_{45}-\mathrm{PIBOA}_{200}$ diblock copolymer nano-objects prepared via thermally initiated PISA at different IBOA concentrations: (a) $15 \% \mathrm{w} / \mathrm{w}$; (b) $30 \% \mathrm{w} / \mathrm{w}$. 

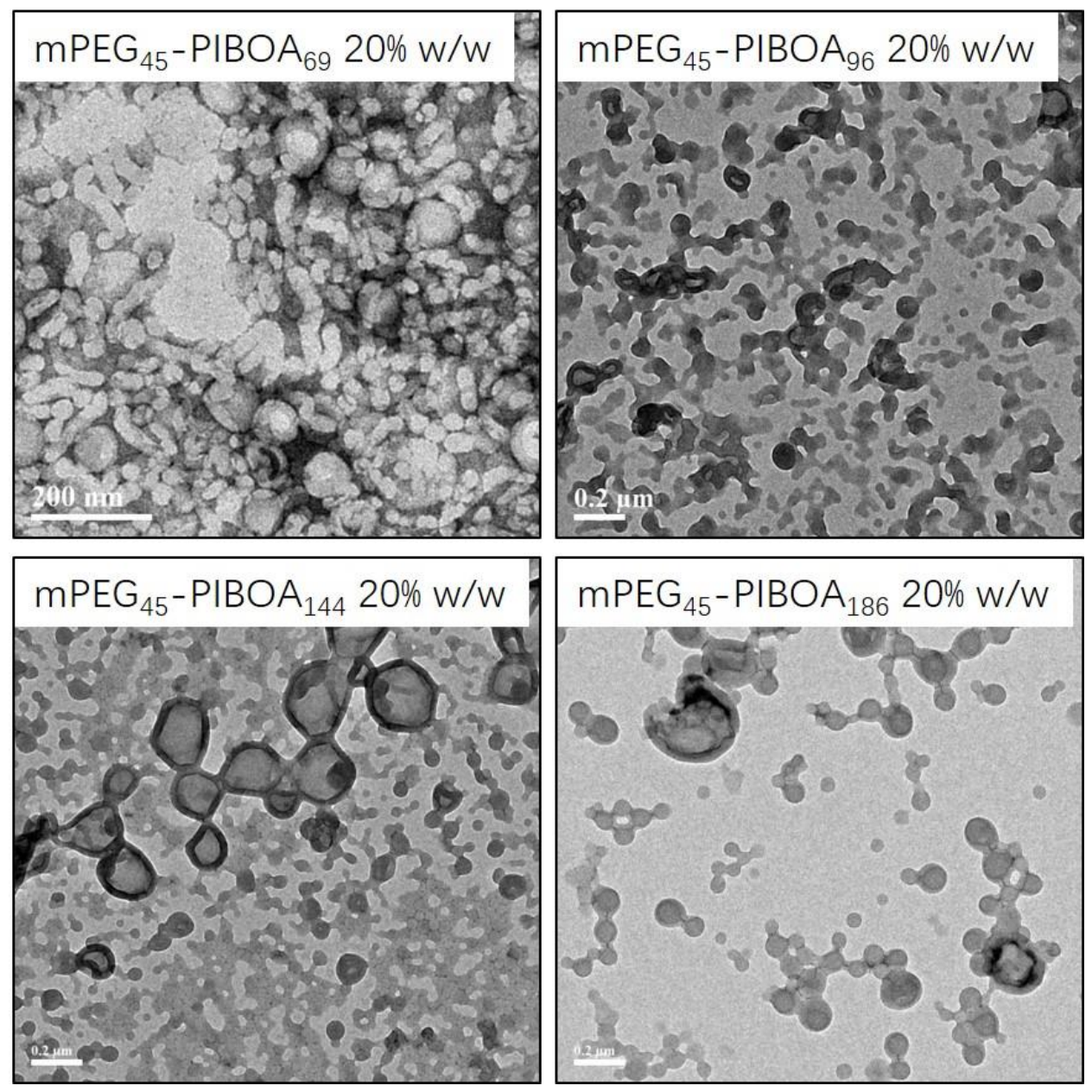

Figure S4. TEM images of $\mathrm{mPEG}_{45}-\mathrm{PIBOA}_{n}$ diblock copolymer nano-objects prepared via alcoholic photo-PISA of IBOA at $20 \% \mathrm{w} / \mathrm{w}$ concentration.

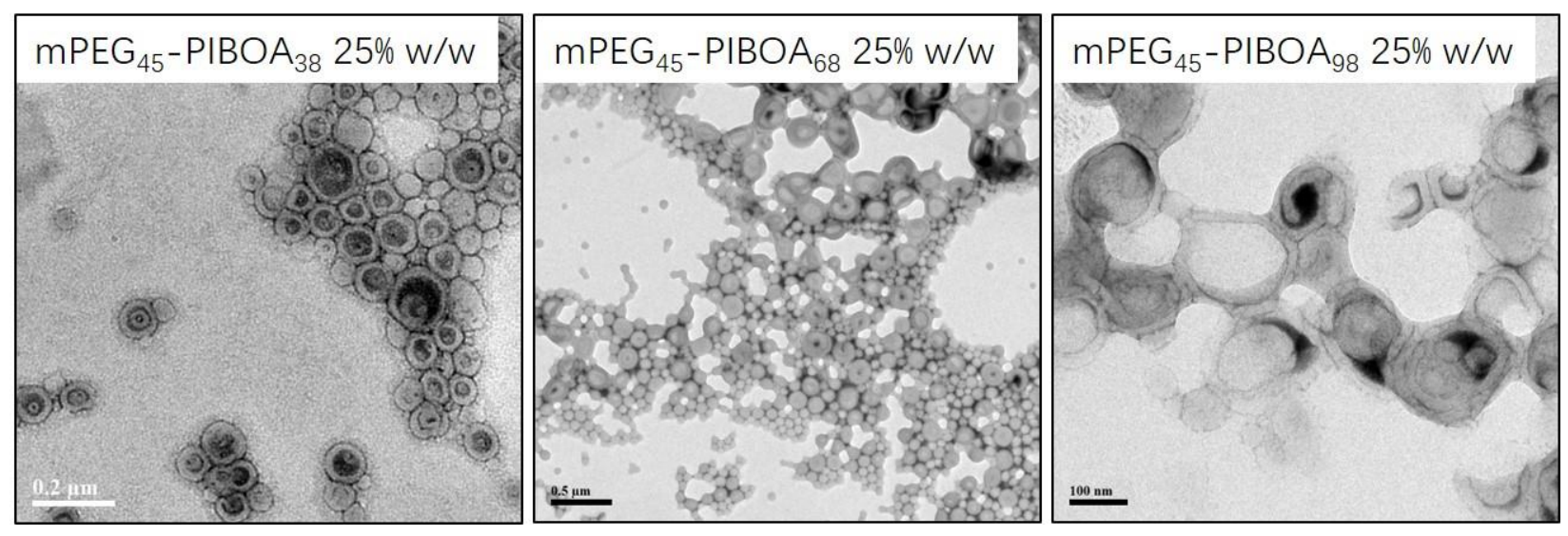

Figure S5. TEM images of $\mathrm{mPEG}_{45}-\mathrm{PIBOA}_{n}$ diblock copolymer nano-objects prepared via alcoholic photo-PISA of IBOA at $25 \% \mathrm{w} / \mathrm{w}$ concentration. 


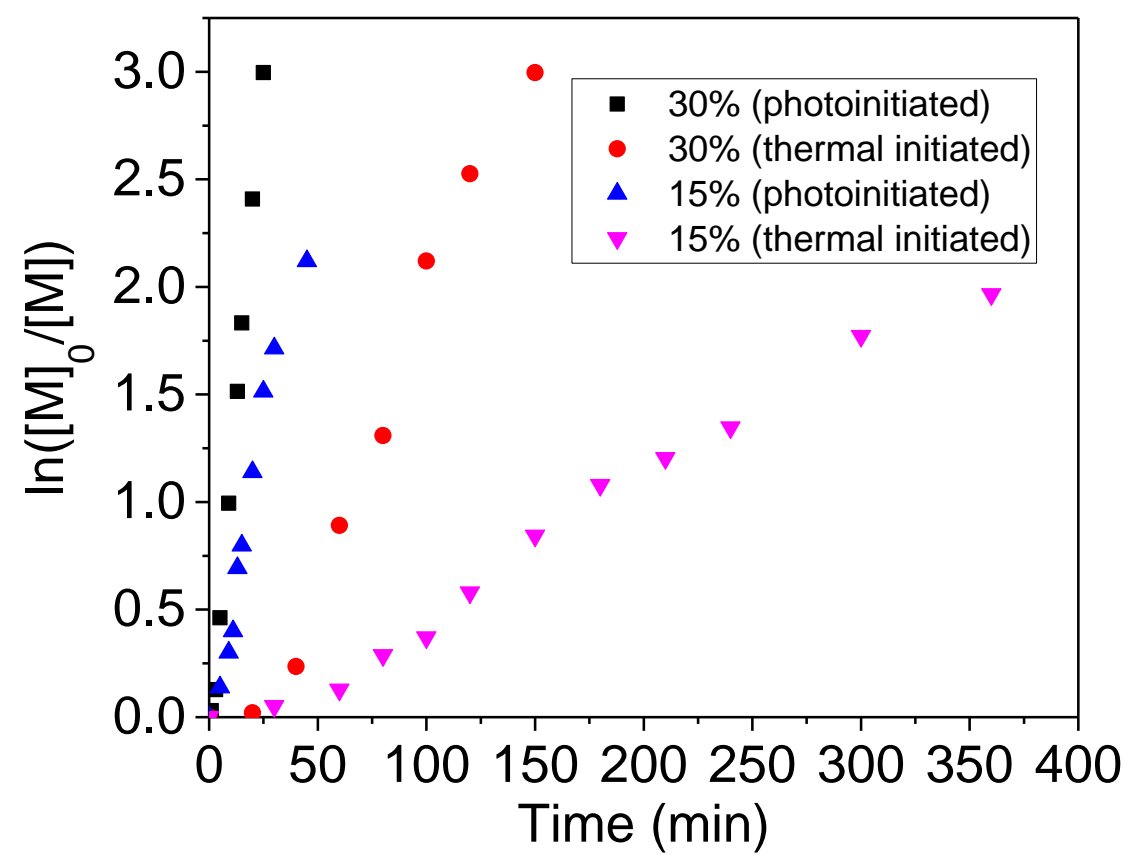

Figure S6. Plots of $\ln ([\mathrm{M}] /[\mathrm{M}] 0)$ versus reaction time for photo-PISA and thermally initiated PISA of IBOA in an ethanol/water mixture at either $30 \% \mathrm{w} / \mathrm{w}$ or $15 \% \mathrm{w} / \mathrm{w}$.

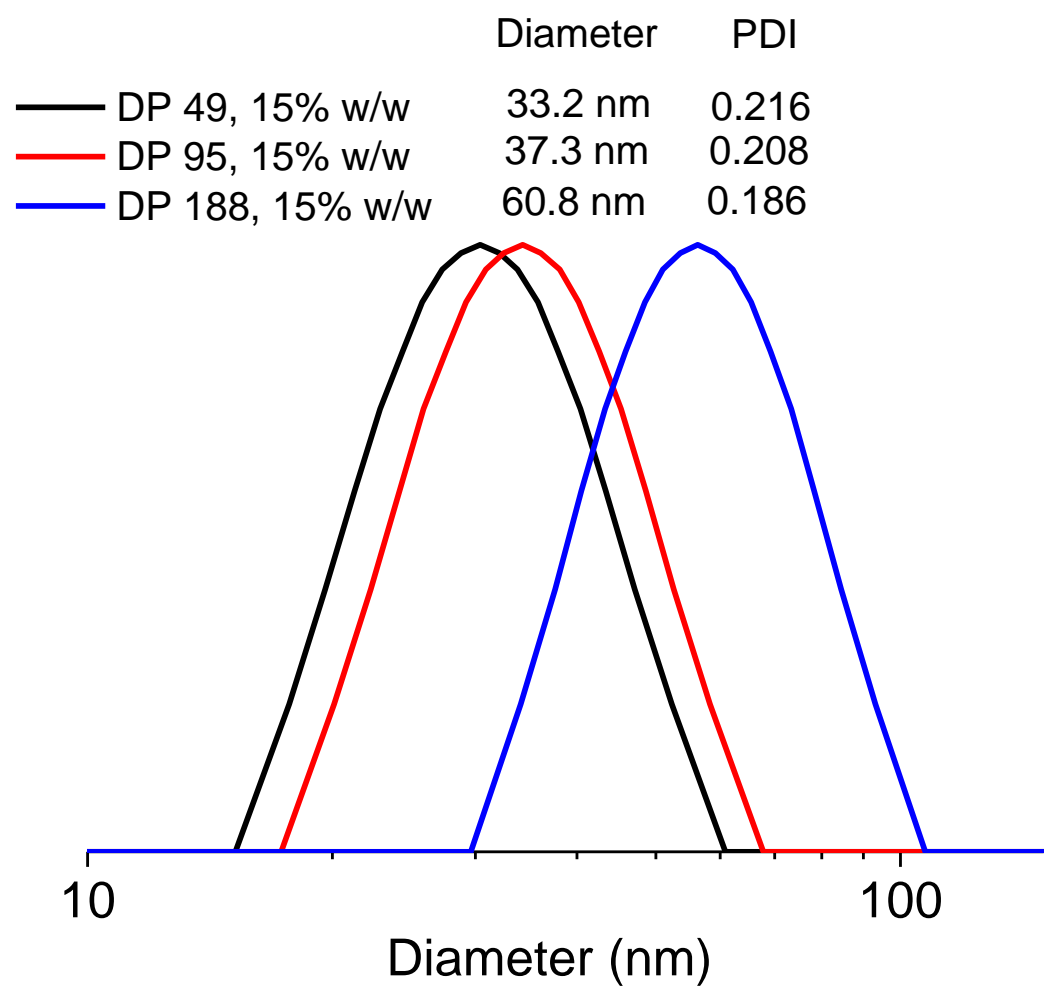

Figure S7. Dynamic light scattering particle size distribution obtained for $\mathrm{mPEG}_{45-} \mathrm{PIBOA}_{n}(\mathrm{n}=49,95,188)$ nanoparticles (prepared at $15 \% \mathrm{w} / \mathrm{w}$ IBOA concentration) 


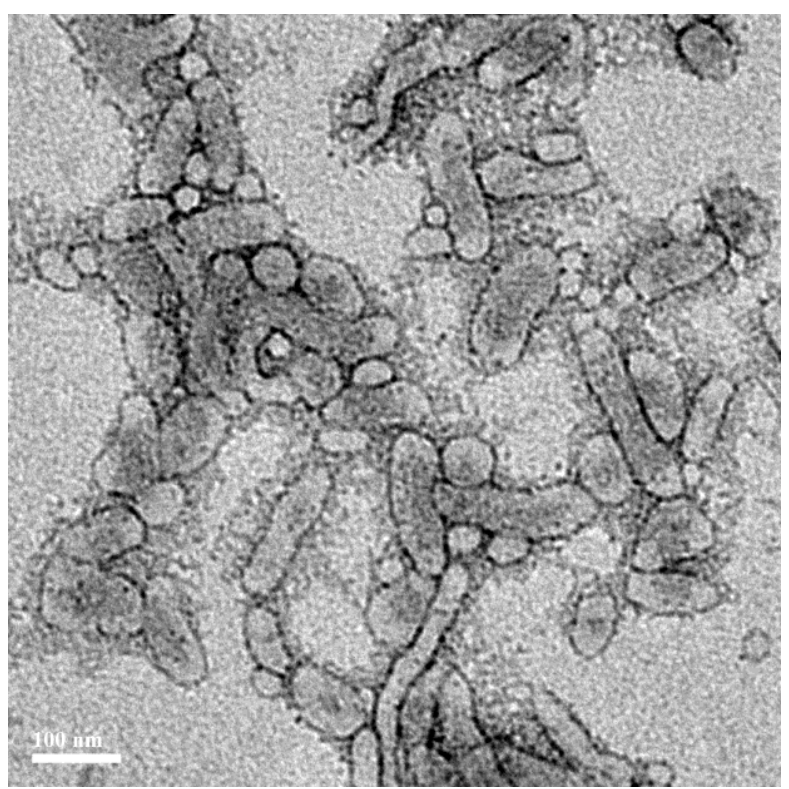

Figure S8. TEM image of $\mathrm{mPEG}_{45}-\mathrm{PIBOA}_{40}$ diblock copolymer nano-objects (prepared at $30 \% \mathrm{w} / \mathrm{w}$ ) after heating to around $70^{\circ} \mathrm{C}$. Note: The TEM sample was prepared at $70^{\circ} \mathrm{C}$.

\section{REFERENCE}

(1) Lai, J. T.; Filla, D.; Shea, R. Macromolecules 2002, 35 (18), 6754-6756. 